\title{
PERFIL DOS RECÉM-NASCIDOS COM DIAGNÓSTICO DE PERSISTÊNCIA DO CANAL ARTERIAL EM UM HOSPITAL TERCIÁRIO DO OESTE PAULISTA
}

\author{
Mary Ellen De Oliveira Martins Disconzi, Murilo Sabbag Moretti
}

Universidade do Oeste Paulista - UNOESTE, Curso de Pós-Graduação em Pediatria, Presidente Prudente, SP. e-mail: maryemartins@hotmail.com

\section{RESUMO}

As deformidades cardíacas congênitas são definidas como uma anormalidade cardiocirculatória estrutural e funcional presente desde o nascimento. O canal arterial é uma estrutura vital para o feto na vida intraútero, e sua abertura persistente após o nascimento pode implicar em serias complicações. Assim, o presente trabalho teve como objetivo caracterizar o perfil dos recém-nascidos com diagnóstico ecocardiográfico de persistência do canal arterial (PCA). Trata-se de um estudo transversal, de cunho retrospectivo, com abordagem quantitativa e analise descritiva de neonatos atendidos em um hospital terciário do Oeste Paulista, realizado no período de janeiro de 2015 a dezembro de 2016. Alguns elementos que se relacionam à PCA como a idade materna ( $>18$ anos), parto cesárea, baixo peso ao nascer, idade gestacional ao nascimento, Apgar no 5ㅇ minuto menor que 7, injúrias hipóxicas e associação com outras cardiopatias foram encontrados nessa casuística.

Palavras-chave: persistência do canal arterial, perfil epidemiológico, neonatos.

\section{PROFILE OF NEWBORNS WITH A DIAGNOSIS OF PERSISTENCE OF THE ARTERIAL CANAL IN A TERTIARY HOSPITAL OF WESTERN SÃO PAULO STATE}

\begin{abstract}
Congenital cardiac deformities are defined as a structural and functional cardiocirculatory abnormality present from birth. The ductus arteriosus is a vital structure for the fetus in intrauterine life, and its persistent opening after birth can lead to serious complications. Thus, the present study aimed to characterize the profile of newborns with an echocardiographic diagnosis of ductus arteriosus persistence (PCA). This is a cross-sectional, retrospective study with a quantitative approach and descriptive analysis of neonates seen at a tertiary hospital in the western of the state, conducted from January 2015 to December 2016. Some elements that relate to PCA such as maternal age ( $>18$ years), cesarean delivery, low birth weight, gestational age at birth, Apgar at $5^{\text {th }}$ minutes less than 7 , hypoxic insults and association with other cardiopathies were found in this series.
\end{abstract}

Keywords: persistence of the ductus arteriosus, profile epidemiological, neonates.

\section{INTRODUÇÃO}

O canal arterial (CA) é uma estrutura presente no feto que comunica a aorta e a artéria pulmonar, desviando o fluxo de sangue do ventrículo direito para circulação sistêmica, já que o feto não utiliza os pulmões intra-útero para hematose, sendo o oxigênio por ele utilizado proveniente da circulação materna, que através da placenta o transfere para o sangue do bebê ${ }^{1}$. Este canal comporta-se como um conduto arterial vital para o adequado desenvolvimento do feto no período gestacional, já que desvia mais da metade do débito cardíaco para a circulação sistêmica e umbílico-placentária. Ao nascimento, ante a elevação sanguínea da pressão de oxigênio, ocorre a constrição desse canal, concomitantemente à dilatação da circulação pulmonar, estabelecendo, dessa forma, um novo padrão circulatório e respiratório no recém-nascido ${ }^{2}$.

O fechamento do CA é inicialmente funcional e após este se torna permanente. No 
recém-nascido a termo o primeiro ocorre com 12 a 15 horas de vida e o segundo com 5 a 7 dias, podendo em alguns casos ser postergado até o 210 dia. Entretanto, nos pré-termo o canal arterial permanece aberto por um período mais prolongado, já que possuem uma maior sensibilidade às prostaglandinas e elevada incidência de hipóxia e acidose neste grupo, além de uma migração deficiente da musculatura que promove a vasoconstricção deste canal ${ }^{1}$. Nesta população a frequência desta cardiopatia é proporcionalmente maior quanto mais imaturo for o neonato, apresentando 58,8\% em recémnascidos com peso inferior a $1000 \mathrm{~g}$ e de $25 \%$ com peso superior a este valor ${ }^{3}$. Especialmente em prematuros, esta anormalidade resulta em alterações hemodinâmicas graves com consequentes morbidades que afetam os sistemas renal, respiratório e gastrointestinal. Desse modo, o reconhecimento precoce desta patologia é de fundamental importância em virtude da implicação prognóstica pela própria deterioração clínica e da sua alta mortalidade, devendo-se implementar o tratamento o mais rápido possível para prevenir o aparecimento de complicações $^{4-6}$.

O exame cardiológico apurado é elemento valioso no diagnóstico de anomalias cardíacas. A suspeita clínica de cardiopatia congênita no período perinatal pode ser levantada pela presença de quatro achados principais: sopro cardíaco, cianose, taquipneia e arritmia cardíaca. No entanto, a avaliação clínica, o eletrocardiograma e a radiografia de tórax são exames complementares de pouca efetividade, tanto no diagnóstico quanto na quantificação da repercussão hemodinâmica da patência deste canal. Por isso o ecocardiograma tem papel fundamental na condução e no diagnóstico dos pacientes com estas características clínicas ${ }^{3}$.

Quando a PCA está acompanhada de sopro cardíaco, aumento da frequência cardíaca, precórdio hiperdinâmico e aumento da amplitude de pulso, a definimos como sintomática, podendo estas manifestações variarem de acordo com a idade gestacional do recém-nascido. Este critério, contudo, não evidencia boa correlação com repercussão hemodinâmica nos primeiros dias de vida, sendo esta determinada pela quantidade de sangue que é desviado da esquerda para a direita pelo canal arterial, gerando hiperfluxo pulmonar e hipofluxo sistêmico, tornando-os susceptíveis a complicações importantes como a broncodisplasia severa ${ }^{1,3}$.

O presente estudo objetiva a

Caracterização do o perfil dos recém-nascidos com diagnóstico ecocardiográfico de persistência do canal arterial no período de janeiro de 2015 a dezembro de 2016, em um hospital terciário do Oeste Paulista.

\section{METODOLOGIA}

Trata-se de um estudo transversal, de cunho retrospectivo, com abordagem quantitativa e analise descritiva. Os dados incluídos foram analisados afim de caracterizar o perfil dos recém-nascidos com diagnóstico ecocardiográfico de PCA atendidos em um hospital terciário do oeste paulista no período de janeiro de 2015 a dezembro do ano de 2016, totalizando 41 crianças. As informações foram obtidas por meio de revisão de 41 prontuários, disponíveis no Serviço de Prontuário do Paciente (SPP), com preenchimento de um roteiro de observação com 12 questões estruturadas, abordando a idade da mãe quando gestante, se realizou ou não o pré-natal, via de parto, doenças maternas adquiridas na gestação, idade gestacional ao nascimento (New Ballard), peso ao nascer, sexo do RN, Escore de Apgar no 1으 e 5은 minuto, necessidade de reanimação em sala de parto, sinais e sintomas relacionados a patologia, idade em que realizou o Ecocardiograma e seu grau de repercussão hemodinâmica.

Para realização do estudo, a pesquisa foi encaminhada e aprovada pelo Comitê de Pesquisa Envolvendo Seres Humanos do hospital e pelo Comitê de Ética em Pesquisa da UNOESTE (CAAE 67573317.4.0000.5515).

O método estatístico empregado foi o descritivo e os resultados apresentados por meio de gráficos e tabelas, contemplando valores absolutos e porcentagens.

\section{RESULTADOS}

Das 194 crianças recém-nascidas atendidas no Serviço de Neonatologia no período de janeiro de 2015 a dezembro de 2016, 41 apresentaram PCA diagnosticado por ecocardiograma. Destes, $87,8 \%$ das mães com crianças acometidas apresentaram idade maior que 18 anos no momento da gestação, sendo que $27 \%$ destas situavam-se no intervalo de $31-35$ anos. 
A realização de pré-natal foi preponderante à sua não realização, sendo que trinta e nove (95\%) das mães em questão referiram frequentar as consultas regularmente. Quanto à via de parto, o mais observado foi a cesariana, alcançando $59 \%$ dos casos estudados (Figura 1).

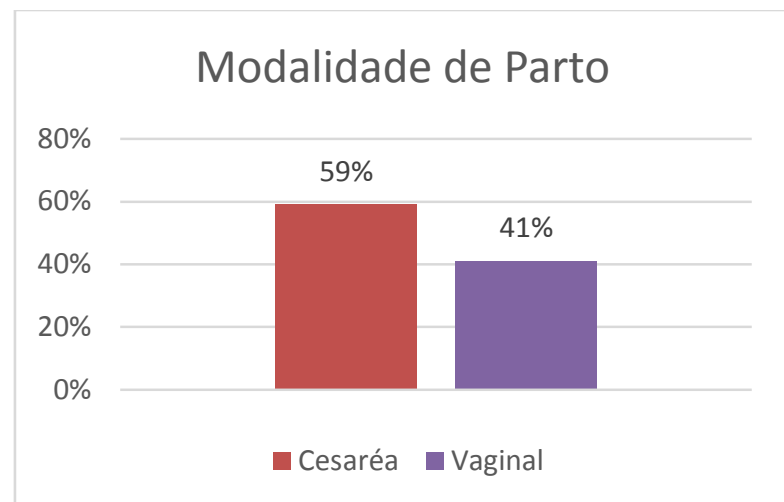

Figura 1. Modalidade de parto dos recémnascidos com diagnóstico ecocardiográfico de PCA.

Cerca de $30 \%$ das mães de crianças com PCA atendidas no Serviço de Neonatologia deste estudo apresentavam patologias durante a gestação, estando a Doença Hipertensiva Específica da Gestação (DHEG) e o Diabetes gestacional responsáveis por $24 \%$ e $5 \%$ dos casos respectivamente (Tabela 1 ).

A idade gestacional no momento do parto é uma variável importante quando nos referimos a uma doença relacionada à prematuridade. Dos casos analisados, a maioria eram prematuros. Onze deles (27\%) eram prematuros extremos, onze (27\%) estavam no intervalo de $28-32$ semanas, treze (32\%) entre 33-36 semanas e apenas seis (14\%) eram recémnascidos a termo, de acordo com o exame físico (New Ballard).

De acordo com o sexo, a PCA foi mais incidente no sexo feminino, contemplando $58,5 \%$ dos casos analisados. Já em relação ao peso de nascimento, grande parte dos bebês atendidos eram de muito baixo peso, perfazendo $68 \%$ do total e $19,5 \%$ de extremo baixo peso. Desse modo, apenas $12 \%$ dos recém-nascidos apresentavam-se com peso superior a $2.500 \mathrm{~g}$ ao nascimento.
Tabela 1. Condições Clínicas maternas de recémnascidos com PCA admitidos na UTI neonatal.

\begin{tabular}{lc}
\hline \multicolumn{1}{c}{ Condição Clínica } & Frequência \\
\hline DHEG & $10(25 \%)$ \\
Diabetes Gestacional & $2(5 \%)$ \\
Outras patologias & $11(27 \%)$ \\
Hígidas & $18(43 \%)$ \\
\hline HEG - Doença hipertensiva específica da gestação.
\end{tabular}

Quanto ás condições de nascimento e apresentação da vitalidade na sala de parto, pelo Escore de Apgar no 10 minuto $39 \%$ situavam-se entre 6-7. Entretanto, no 50 minuto $61 \%$ já apresentavam-se com Apgar $>7$. Vinte e três dos neonatos foram submetidos à reanimação logo após o nascimento, dentre os quais $61 \%$ receberam 2 ciclos de ventilação com pressão positiva (VPP), duas evoluíram para intubação orotraqueal e $3 \mathrm{com}$ parada cardiorrespiratória com uso de Adrenalina (Figura 2).

Dentre os sintomas que podem vir acompanhados da persistência do canal arterial, foram observados 16 (39\%) recém-nascidos com sopro cardíaco e 13 (32\%) com taquicardia, estando muitas vezes sobrepostos (32\%).

O diagnóstico ecocardiográfico da patologia estudada foi realizado em $53,6 \%$ dos recém-nascidos após o 70 dia de vida e antes disso em 46,4\%, evidenciando repercussão hemodinâmica em $34 \%$ no total de casos.

\section{DISCUSSÃO}

Dos 45 recém-nascidos com PCA diagnosticadas por ecocardiograma no Serviço de Neonatologia no período de janeiro de 2015 a dezembro de 2016, 41 prontuários foram analisados, revelando que a grande maioria das mães no momento do parto eram maiores de idade e $27 \%$ destas situavam-se no intervalo de 31-35 anos, com risco maior para gerar crianças portadoras de síndromes genéticas, que podem cursar com maior prevalência de cardiopatias congênitas segundo Santos et al. ${ }^{7}$ Relacionando ao gênero, encontramos uma incidência maior no sexo feminino, contemplando $58,5 \%$ dos casos analisados, compatível com o encontrado em estudo de Huber et al. ${ }^{8}$. 
Reanimação Neonatal na Sala de Parto

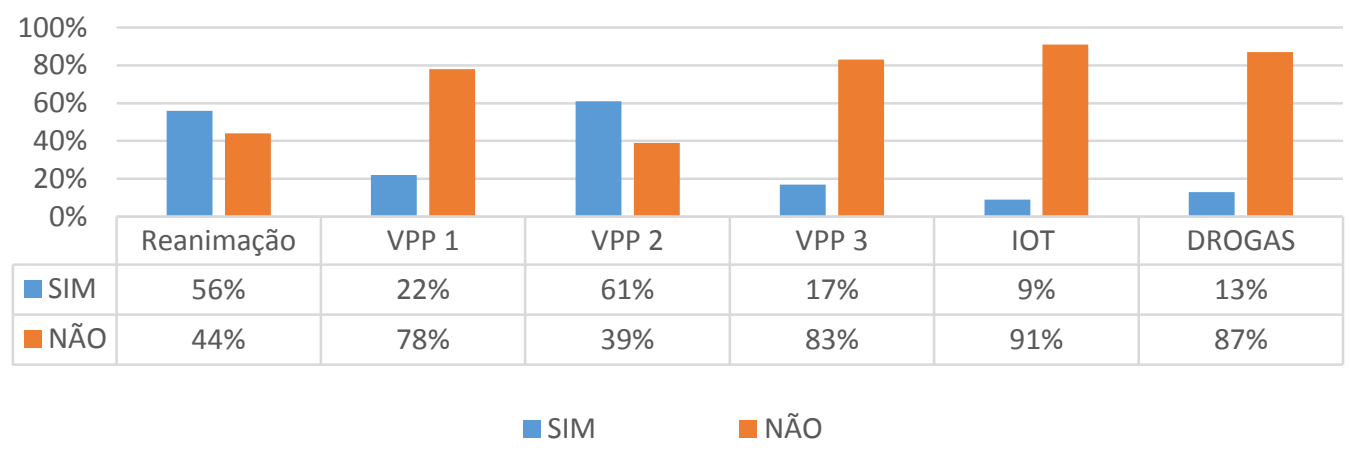

Figura 2. Recém-nascidos com PCA que necessitaram de reanimação neonatal na sala de parto.

Quanto à assistência pré-natal, 95\% das mães a realizaram, e cerca de $60 \%$ tiveram seus filhos por parto cesárea, sendo que patologias maternas estavam envolvidas em $30 \%$ do total de neonatos avaliados. Nesse contexto, Huber et al. ${ }^{8}$ inferem que a presença de doenças maternas assim como uso de medicações e drogas, podem levar a doenças cardíacas congênitas.

A PCA é uma anomalia que acomete grande número de RN prematuros, sobretudo naqueles com baixo peso, podendo levar a repercussões clínicas significativas, com impacto na morbimortalidade desses pacientes. Isso vai de encontro ao observado neste trabalho, onde apenas $14 \%$ dos neonatos estudados eram a termo pelo exame físico do recém-nascido (New Ballard). Diante disso, sabe-se que a prevenção da prematuridade está intimamente relacionada a uma diminuição da incidência de patência do canal arterial, bem como de suas complicações como broncodisplasia pulmonar e enterocolite necrotizante. De acordo com Miyague ${ }^{3}$, esta seria mais uma consequência da prematuridade, já que possuem uma maior sensibilidade às prostaglandinas e elevada incidência de hipóxia e acidose, mantendo o canal aberto por um tempo mais prologado.

A frequência desta cardiopatia é proporcionalmente maior quanto mais imaturo for o neonato, e consequentemente ao baixo peso apresentado por eles. No estudo de Belo et al. ${ }^{9}$, mais da metade dos prematuros com peso abaixo de $1.500 \mathrm{~g}$ apresentaram algum tipo de cardiopatia, entre elas a PCA, o que podemos observar também em nossa casuística, onde aproximadamente $45 \%$ dos casos revisados apresentavam peso $<1.500 \mathrm{~g}$. Esse mesmo autor destaca ainda que quanto menor o peso de nascimento, maiores as chances de doenças cardíacas. Nossos resultados se assemelham, pois somente $12 \%$ dos recém-nascidos com esta cardiopatia apresentavam-se com peso superior a $2.500 \mathrm{~g}$ ao nascimento.

Santos et al. ${ }^{7}$ descrevem uma associação entre a presença de anomalias congênitas e as variáveis: menor duração da gestação, menor escore de Apgar no quinto minuto, baixo peso ao nascimento e tipo de parto (cesáreo). Esta relação pode ser vista em nossos dados, já que apenas $22 \%$ dos pacientes com PCA apresentaram Apgar $>7$ no primeiro minuto, contudo, no 50 minuto $61 \%$ já apresentavam-se com pontuação acima dessa cifra. Outra variável observada foi a necessidade de reanimação (56\%) e de ventilação mecânica invasiva (9\%) em sala de parto nesses pacientes. Visconti et al. ${ }^{1}$ salientam que injúrias sofridas pelos neonatos como hipóxia e infecções levam a liberação de mediadores inflamatórios, os quais dificultariam o fechamento espontâneo do CA, aumentando a incidência desta anomalia.

O diagnóstico precoce das anomalias cardíacas congênitas é importante ferramenta para uma boa evolução clínica dos pacientes e prevenção de maiores prejuízos e agravos à saúde dos mesmos. Diante da suspeição clínicoepidemiológica, logo deve se realizar o ecocardiograma, um exame indispensável na confirmação diagnóstica e na decisão terapêutica ${ }^{7}$. No hospital em que se baseou este estudo, o diagnóstico ecocardiográfico da PCA foi realizado em $53,6 \%$ dos recém-nascidos após o 70 dia de vida e antes disso em $46,4 \%$. Destes $34 \%$ tinham repercussão hemodinâmica, e foram precocemente tratados. 


\section{CONCLUSÃO}

Com este trabalho foi possível caracterizar alguns elementos que se relacionam à PCA, em consonância com a literatura, dentre eles: idade materna (>18 anos), parto cesárea, baixo peso ao nascer, idade gestacional ao nascimento, Apgar no 50 minuto menor que 7, injúrias hipóxicas e associação com outras cardiopatias. O diagnóstico só é feito quando há alteração no exame clínico, devendo bem executado, ou quando o neonato está incluído em um grupo predisposto, como é o caso de prematuros, baixo peso ao nascer, uso de drogas e doenças maternas, e injúrias no periparto, sendo o diagnóstico ecocardiográfico. Muitas vezes não se encontram alterações ao exame físico ou estas são muito sutis, fazendo com que o pediatra deva conhecer o perfil epidemiopatológico dos bebês que possam vir apresentar esta afeç̧ão para levantar esta hipótese e prover sua investigação.

\section{CONFLITO DE INTERESSES}

Os autores declararam não haver qualquer potencial conflito de interesse que possa interferir na imparcialidade deste trabalho cientifico.

\section{REFERÊNCIAS}

1. Visconti LF, Morhy SS, Deutsch AD, Tavares GMP, Wilberg TJM, Rossi FS. Características clínicas e ecocardiográficas associadas à evolução do canal arterial em recém-nascidos com peso de nascimento inferior a 1.500g. Einstein (São Paulo). 2013;11(3):31723. DOI: $\quad$ https://doi.org/10.1590/S1679$\underline{45082013000300010}$

2. Locali RF, Matsuoka PK, Gabriel EA, Bertini JA, La Rotta CA, Catani R et al. Tratamento da persistência de canal arterial em recém-nascidos prematuros: análise clínica e cirúrgica. Arq Bras Cardiol. 2008;90(5):345-49. DOI: $\quad$ https://doi.org/10.1590/S0066$\underline{782 \times 2008000500007}$

3. Miyague NI. Persistência do canal arterial em recém-nascidos prematuros. J. Pediatr. 2005; 81(6):429-30. DOI: https://doi.org/10.2223/JPED.1415

4. Nina RVAH, Gama MEA, Santos AM, Nina VJS, Figueiredo Neto JA, Mendes VGG et al. O escore de risco ajustado para cirurgia em cardiopatias congênitas (RACHS-1) pode ser aplicado em nosso meio?. Rev Bras Cir Cardiovasc. 2007;22(4): 425-31. DOI: $\quad$ https://doi.org/10.1590/S0102$\underline{76382007000400008}$
5. Costa RN, Pereira FL, Ribeiro MS, Pedra SRF, Succi F, Marques $P$ et al. Tratamento percutâneo vs. cirúrgico da persistência do canal arterial em crianças e adolescentes. Rev Bras Cardiol Invasiva. 2012;20(3):315-23. DOI: https://doi.org/10.1590/S2179-83972012000300016

6. Pacifici GM. Ibuprofen and indomethacin for the closure of the patent ductus arteriosus. Med Express. 2016;3(3):M160301. DOI: https://doi.org/10.5935/MedicalExpress.2016.03.01

7. Santos ADS, Menezes GA, Sousa DS. Perfil dos recém-nascidos com cardiopatia congênita em uma maternidade de alto risco do município de Aracaju. Cad Grad - Ciênc Biol Saúde. 2013;1(17):59-70.

8. Huber J, Peres VC, Santos TJ, Beltrão LF, Baumont $A C$, Cañedo $A D$ et al . Cardiopatias congênitas em um serviço de referência: evolução clínica e doenças associadas. Arq Bras Cardiol. 2010;94(3):333-8. DOI: https://doi.org/10.1590/S0066-782X2010000300009

9. Belo WA, Oselame GB, Neves EB. Perfil clínicohospitalar de crianças com cardiopatia congênita. Cad Saúde Colet. 2016;24(2):216-20. DOI: https://doi.org/10.1590/1414-462X201600020258

Recebido para publicação em 08/08/2017

Revisado em 19/09/2017

Aceito em 20/09/2017 